\title{
Exosomal Telomerase Transcripts Contribute to CAF Formation and Reprogram the MicroRNA Transcriptome Profile of the Recipient Cells
}

\section{Daniela Likonen}

The Felsenstein Medical Research Center

Maria Pinchasi

The Felsenstein Medical Research Center

Einat Beery

The Felsenstein Medical Research Center

Roded Sharan

School of Computer Science, Tel-Aviv University

Asia Gervits

School of Computer Science, Tel-Aviv University

Lorenzo Signorini

School of Computer Science, Tel-Aviv University

Meir Lahav

Sackler School of Medicine, Tel-Aviv University

\section{Pia Raanani}

Institute of Hematology, Davidoff Cancer Center, Rabin Medical Center

Orit Uziel ( $\nabla$ oritu@clalit.org.il )

The Felsenstein Medical Research Center

\section{Research Article}

Keywords: Human telomerase reverse transcriptase (hTERT), exosomes, microRNA, tumor microenvironment, cancer

Posted Date: November 16th, 2021

DOI: https://doi.org/10.21203/rs.3.rs-1026385/v1

License: (c) (1) This work is licensed under a Creative Commons Attribution 4.0 International License.

Read Full License 


\section{Abstract}

It is now well accepted that cancer cells change their microenvironment from normal to tumor-supportive state to provide sustained tumor growth, metastasis and drug resistance. These processes are partially carried out by exosomes, nano-sized vesicles secreted from cells, shuttled from donor to recipient cells containing a cargo of nucleic acids, proteins and lipids. By transferring biologically active molecules, cancer-derived exosomes may educate microenvironmental cells to become tumor supportive. We have recently shown that the transcript of human telomerase reverse transcriptase (hTERT), the hallmark of cancer cells is packaged in cancer cells derived- exosomes. After the engulfment of the hTERT transcript into fibroblasts, it is translated into a fully active enzyme. Telomerase activity in the recipient, otherwise telomerase negative cells, provides them with a survival advantage. Here we show that exosomal telomerase plays a role in modifying normal fibroblasts into cancer associated fibroblasts (CAFs) by upregulating aSMA and Vimentin, two CAF markers. We also show that telomerase activity changes the transcriptome of microRNA in these fibroblasts. By ectopically expressing microRNA 342, one of the top identified microRNAs we show that it may mediate the proliferative phenotype that these cells acquire upon taking-up exosomal hTERT, providing them with a survival advantage.

\section{Introduction}

The importance of the microenvironment for the development, perpetuation and spreading of the tumor mass has been extensively shown $[1,2]$. Secreted from most cell types, including cancer cells, exosomes are nanosized particles (30-150 in diameter) that mediate the cross talk between environmental and tumor cells. Exosomes carry a cargo of nucleic acids, proteins and lipids which reflects the cellular content of their cells of origin. They travel in all body fluids and are taken up by cells in their vicinity. By transferring biologically active molecules to these recipient cells, cancer derived exosomes may educate microenvironmental cells to become tumor supportive cells, thus acting as intercellular communicators [3].

Telomerase, a reverse transcriptase which elongates telomeres, is a hallmark of cancer cells as it is expressed in the vast majority of malignant cells and is repressed in most somatic cells. By preventing telomeres' shortening, it provides cancer cells with endless replicative potential [4]. We have recently shown that exosomes derived from cancer cells contain the transcript of telomerase. When these exosomes were taken up by fibroblast, otherwise telomerase negative cells, the transcript was translated into a fully active enzyme, transforming these cells into telomerase positive cells, providing them with a survival advantage [5]. Apart from telomere synthesis, telomerase possess other "extracurricular" activities, which are telomere length independent, for example, prevention of apoptosis [6]. Another example is its involvement in microRNA upregulation [7]. The goal of the current study was to explore whether some of the phenotypic changes carried out by exosome-derived telomerase activity in the fibroblast cells were mediated by modulating these cells into cancer associated fibroblasts (CAFs) and by the upregulation of specific microRNAs in these cells. 


\section{Materials And Methods}

\section{Cell lines, growth conditions and cell's transfection}

The following cell lines were used in the study: Acute T cell leukemia (Jurkat, kindly provided by Dr. Galit Granot, FMRC), primary human foreskin fibroblast (pHFF, kindly provided by Dr. Selig from the Israel Institute of Technology); human foreskin fibroblast (hFF). The hFF cell line was immortalized by the ectopic expression of hTERT as previously described [8] and kindly provided by Dr. Selig from the Israel Institute of Technology.

Jurkat cell line was cultured inRPMI-1640 with $20 \%$ fetal bovine serum (FBS), containing 100 units/ml LGlutamine and 1\% streptomycin (Biologicallndustries Beit Haemek, Israel).hFF andpHFF cells were cultured in Dulbecco's minimal essential medium (DMEM) with 20\% FBS containing 100 units $/ \mathrm{ml}$ LGlutamine and $1 \%$ streptomycin (Biologicallndustries). The cells were incubated at $37^{\circ} \mathrm{C}$ in $5 \% \mathrm{CO}$. For exosome isolation an exosome free media was prepared by ultracentrifugation of FBS at $100,000 \mathrm{~g}$ for 16 hours at $4^{\circ} \mathrm{C}\left(\right.$ Optima $^{\mathrm{TM}} \mathrm{XPN}$, Beckman coulter, USA).

For cell transfection,2.5pHFF cells/well were seeded in 6-well plate for $24 \mathrm{hr}$ and were subsequently transfected by using HiPerFect transfection reagent (Qiagen, Germany) according to the manufacturer's instructions. Briefly, miRNA mimics was diluted (150 ng) in $100 \mu$ of culture medium without serum for a final concentration of $5 \mathrm{nM}$. Then it was mixed with $12 \mu \mathrm{l}$ of HiPerFect transfection reagent to allow the formation of transfection complexes in room temperature for $10 \mathrm{~min}$. The resulting transfection solutions were then added to each well containing $2.3 \mathrm{ml}$ of culture medium. Cells were grown at $37^{\circ} \mathrm{C}$ for $72 \mathrm{~h}$ before another transfection was performed as above.

Cells were transfected with the following miScript miRNAs:

- hsa miR-342-3p miRNA (miR342 mimic)

- miRNA Negative Control with a scrambled sequence.

- miRNA Inhibitor

- miRNA Inhibitors Negative Control

\section{Isolation of exosomes}

Exosomes were isolated by serial ultracentrifugation as previously described [9]. Briefly, a total of $5 x$ Jurkat cells were grown in exosome depleted media for 3 days. Then, the cells debris was removed by 2 consecutive centrifugations at 1,000 RCF 2,000 RCF each. Subsequently, the media was centrifuged at $10,000 \mathrm{RCF}$ for $30 \mathrm{~min}$ at $4^{\circ} \mathrm{C}$ and the supernatant collected and filtered through $0.22 \mu \mathrm{m}$ filters (Merck Millipore, USA). The clarified medium was than centrifuged at $100,000 \mathrm{RCF}$ at $4^{\circ} \mathrm{C}$ for 70 minutes (Beckman Coulter, CA, USA). The crude exosome-containing pellets were resuspended in $1 \mathrm{~mL}$ and a second round of ultracentrifugation at $100,000 \mathrm{RCF}$ at $4^{\circ} \mathrm{C}$ for 120 minutes was carried out. The resulting 


\section{Nanosized tracking analysis of Jurkat derived exosomes}

Exosomes were quantified by using the Nanosight tracking analysis, according to the manufacturer's instructions (Malvern Panalytical, Cambridge UK) and following published method [10]. The analysis settings were optimized and kept constant between samples.

\section{BCA Protein quantification}

Exosomes were suspended in RIPA buffer and centrifuged for protein extraction. Total protein was quantified using Pierce BCA Protein Assay Kit (Thermo Scientific, MA, USA) in accordance with the manufacturer's instructions.

\section{Exposure of fibroblasts to Jurkat derived exosomes}

$200 \mu$ ls of the resuspended exosomes were added to $3 \times 10^{5}$ pHFF cells per well in a 6 -well culture plate. 6 hours later the fibroblast cells were harvested and RNA was extracted for the measurement of hTERT expression by q-PCR. Previous studies conducted in our laboratory have suggested that exosomes affected the recipient cells in a dose and a time dependent manner. Therefore, we used these conditions in all further experiments.

\section{RNA isolation}

Total RNA was extracted using the EZ-RNA II Isolation Kit reagent (Biological Industries Beit Haemek, Israel) according to manufacturer's instructions. Briefly, cells were lysed with guanidine thiocyanate detergent solution, followed by organic extraction and alcohol precipitation of the RNA. Quantification and the purity of total RNA were assessed by using the NanoDrop $1000 \AA$ spectrophotometer (Thermo Fisher Scientific, MA, USA).

RNA isolation from exosomes was performed by using Total Exosome RNA and Protein Isolation Kit (Invitrogen, CA, USA). Exosomes were lysed with denaturing solution and then precipitated by acidphenol: chloroform solution followed by several washes and centrifugation steps. The supernatant was then placed on filter cartridge tubes for further purification using numerous washing solutions provided by the kit. RNA was eluted with PCR grade water.

\section{cDNA formation}


Total RNA extracts from cells were reverse transcribed using the High-Capacity cDNA Reverse Transcription Kit (Applied Biosystem, CA, USA) according to the manufacturer's instructions. Briefly, $1,000 \mathrm{ng}$ of template RNA was added to $10 \mu \mathrm{L}$ reaction mixture containing dNTP's, random primers, RNase inhibitor, reaction buffer and reverse transcriptase. The incubations steps included $10 \mathrm{~min}$ at $25^{\circ} \mathrm{C}$ followed by $120 \mathrm{~min}$ at $37^{\circ} \mathrm{C}$ and the reaction was terminated by a heating step at $85^{\circ} \mathrm{c}$ for $5 \mathrm{~min}$.

\section{Q-real time PCR reaction}

Real-time-PCR was employed to estimate the levels of the hTERT mRNA in exosomes secreted from the growth media of Jurkat cells based on the Taqman methodology (Applied Biosystems, CA, USA) and for the validation of the results of the microRNAs analysis. The expression of the HPRT-1 (Hypoxanthineguanine-phosphoribosyltransferase-1) gene was used as the endogenous control. PCR reactions were carried out using the Step One Plus detection system (Applied Biosystems, CA, USA). Taqman real time PCR reactions were performed with 50ng of $C D N A$, specific primers and Taqman gene expression master mix (Applied Biosystems, CA, USA). The incubations steps included 2 min at $50^{\circ} \mathrm{C}$ followed by $10 \mathrm{~min}$ at $95^{\circ} \mathrm{C}$ and 40 cycles of 15 seconds at $95^{\circ} \mathrm{C}$ and $1 \mathrm{~min}$ at $60^{\circ} \mathrm{C}$.

PCR reactions were prepared with the following custom made Taqman fluorochrome labelled primers (Applied Biosystems, CA, USA).

hTERT primers: Forward: 5'-CGTCCAGACTCCGC TTCATC-3'

Reverse: 5'-GAGACGCTCGGCCCTCTT-3';

HPRT-1 primers: Forward: 5-'TTATGGACA GGACTGAACGTCTTG-3'

Reverse: 5'-TGTAATCCAGCAGGTCAGCAAA-3'

\section{Western blot}

The expression of CAFs markers in exosomal exposed fibroblast cells was assessed by Western immunoblot analysis. Proteins were extracted and quantified using the Bradford assay (Bio-Rad Laboratories, CA, USA). $25 \mu \mathrm{g}$ of proteins extracted from treated and untreated cells were subjected to Poly-Acrylamide Gel Electrophoresis and transferred to a nitrocellulose membrane. Membrane was hybridized with antibodies against the different proteins: aSMA (1:500, Bio-Rad, California, USA); Vimentin (1:200, Santa Cruz Biotechnology, Texas, U.S.A.); and subsequently to fluorescent labeled secondary antibodies. Visualization was done by the Odyssey analysis software (Odyssey IR imaging system; LI-COR).

\section{ELISA assay}


The expression of IL- 6 by exosome exposed fibroblast cells was evaluated by ELISA assay. Culture supernatants were collected centrifuged to pellet any detached cells and analyzed using a Human IL-6 Quantikine ELISA Kit (R\&D Systems Inc., MN, USA) according to the manufacturer's instructions. Culture supernatants were diluted 150-1000-fold for IL-6 measurements.

\section{miRNA profiling}

microRNA expression levels were determined by using the Rosetta Genomics proprietary microarrays platform containing 2167 microRNA probes and various control probes. RNA was extracted and labeled; samples were processed according to standard protocols. Data was stored in the Microarray Database. Statistical probability of significance (adjusted $p$ values, set at $p<0.05$ ) and a fold change of $>2$ were used to select the miRNAs that were significantly differentially expressed between our samples. The miRBase website [12] was used to retrieve the mature sequences for each miR.

\section{Validation of the results of the microRNA analysis}

q-PCR was performed to validate and quantify the expression of the results of the microRNA analysis (miR-342-3p, miR-125b-1, miR-128-3p and miR-92a-3p). Prior to RNA isolation as described above, pHFF cells were exposed to Jurkat cells derived exosomes for $6 \mathrm{~h}$ and the levels of microRNA were determined by Q-PCR. pHFF cells without exosome exposure served as controls. Specific commercial primers sequences were used for the validation of each miRNAs ( $A B I, F o s t e r$ City, $C A, U S A)$.

\section{miRNA cDNA formation}

For miRNA-specific cDNA formation we used 20ng total RNA from the RNA extraction samples. Taqman ${ }^{\circledR}$ small RNA Assay was used for miRNA reverse transcription reaction. 1-10 ng of total RNA sample were used per $15 \mu \mathrm{l}$ RT reaction. Taqman ${ }^{\circledR}$ MicroRNA probe was used as the Reverse Transcription primers. Each reaction included $7 \mu \mathrm{l}$ master mix, $3 \mu \mathrm{l} 5 \mathrm{X}$ RT primers (miRNA specific primers) and $5 \mu \mathrm{l}$ of RNA sample (1-10 ng).

\section{miRNA Q-real time PCR reaction}

Taqman ${ }^{\circledR}$ Small RNA Assays kit was used for Real-time quantitative PCR according to the provided manual $(A B I, C A, U S A)$, run and analyzed by the Step- One Q-PCR device(ABI).

miRNA specific primers were chosen for the following miRNAs: miR-342-3p, miR-125b-1, miR-128-3p and miR-92a-3p. U3 was selected as a housekeeping gene to normalize the miRNA levels. 
The incubation steps included: $10 \mathrm{~min}$ at $95^{\circ} \mathrm{C}$, followed by 40 cycles of 15 seconds at $95^{\circ} \mathrm{C}$ and 60 seconds at $60^{\circ} \mathrm{C}$.

\section{Proliferation assay}

Cellular proliferation of pHFF cells was assessed by the Sulphorodamine B (SRB) assay. Post transfection, cells $/ \mathrm{ml}$ were cultured in 24 -well plates for $72 \mathrm{~h}$. Cells were then fixated to the plate with $10 \%$ Tetrochloric Acid and incubated at $4^{0} \mathrm{C}$ for $1 \mathrm{~h}$, then washed with $\mathrm{dH}_{2} \mathrm{O}$. Subsequently, $1 \mathrm{ml}$ of SRB coloring agent (Sigma Aldrich Israel Ltd., Rehovot, Israel) was added to the wells which were incubated at roomtemperature for 30 mins and then washed with $1 \%$ acetic acid. After adding $10 \mathrm{nM}$ TRIS.HCl buffer absorbance was measuredat $515 \mathrm{~nm}$ by an ELISA microplate reader (Biotek InstrumentsInc., VT, USA). Experiments were performed in triplicates.

\section{Cell cycle analysis}

The cell cycle status was analyzed by flow cytometry. Cells were processed by standard methods using propidium iodide staining of the DNA. Briefly, after cells' transfection upon reaching $60 \%$ confluence, the cells were trypsinized, resuspended in $5 \mathrm{ml}$ cold PBS and centrifuged at $2,500 \mathrm{rpm}$ for 5 mins at $4^{\circ} \mathrm{C}$. Cells were subsequently pelleted and fixed by adding $4.5 \mathrm{ml} 70 \%$ cold ethanol dropwise to 0.5 cold PBS, with gentle mixing. The cells were then incubated for $>2 \mathrm{~h}$ at $-20^{\circ} \mathrm{C}$. Cells were subsequently washed by PBS and centrifuged at 2,500 rpm for 5 minutes $4^{\circ} \mathrm{C}$. Staining with propidium iodide (P4170, Sigma Aldrich) was conducted prior to flow cytometry analysis. Cells were pelleted and resuspended in $0.5 \mathrm{ml}$ PBS

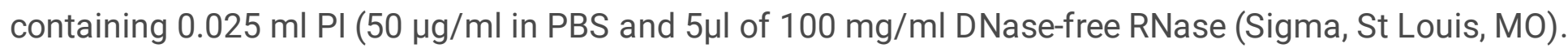
Cells were then incubated at $37^{\circ} \mathrm{C}$ for 15 minutes. Samples were analyzed on a Beckman-Coulter Epics $\mathrm{XL}-\mathrm{MCL}$ apparatus. The parameters were adjusted for the measurement of single cells using the forward and side scatter plots.

\section{Prediction of miRNA targets}

To determine potential mRNA targets for specific miRNAs we have used several publicly available target prediction algorithms based on sequence complementarity between the miRNA and its potential mRNA target $3^{\prime}$ untranslated region (3'UTR).

TargetScan[11] was used to determine potential miR-342 targets involved in cell proliferation and cellcycle. In addition to TargetScan, a combination of bioinformatics tools for miRNA target prediction, includingmiRBase [12],miRanda [13], DIANAmt [14], miRDB [15], miRWalk [16] and PITA [17] were used.

\section{Functional annotation and gene set enrichment analysis}


Several bioinformatic tools have been used for the biological interpretation and the analysis of pathways associated with defined lists of genes. These included DIANA miRPath and DAVID. mirPath is a webbased computational tool developed to identify molecular pathways potentially altered by the expression of single or multiple microRNAs. The software performs an enrichment analysis of multiple microRNA target genes comparing each set of microRNA targets to all known pathways. Pathways with p-value $<0.05$ providing statistically significant values are selected [18]. mirPath was used to gain insight into global molecular networks and canonical pathways specific to miR-342.

The Database for Annotation, Visualization and Integrated Discovery (DAVID) v6.7 bioinformatic algorithm [19] was used for the functional annotation analysis of Gene Ontology (GO) terms associated with a given gene list of confirmed mRNA targets within each miRNA cluster specific for miR-342. We analyzed the list of the most significantly regulated genes using the ClueGO Cytoscape plugin [20]. GO functional categories with $p$-values under 0.05 were considered statistically significant. To study the putative interactions between the four selected miRs and telomerase, we identified proteins that are upstream to these miRs. These proteins were subjected to the Advanced Network Analysis Tool (ANAT) software, a tool for constructing and analyzing functional protein networks. ANAT provides access to upto-date large-scale physical association of protein data, advanced algorithms for network reconstruction and several tools for exploring and evaluating the obtained network models. We used the network-based analysis inferring an anchored network (telomerase) that connects a given set of proteins (proteins that regulate the four selected miRs expression) to designated anchor set of proteins [21].

\section{Statistical analysis}

Quantitative data were expressed as means \pm SD. Statistical significance was determined by the Student's t-test. P-value $<0.05$ was considered as statistically significant.

\section{Results}

\section{Jurkat cells derived exosomes are taken up by fibroblast cells}

To harvest Jurkat cells derived exosomes we grew the cells in exosomes free media for $72 \mathrm{~h}$. Exosomes were isolated using differential centrifugation. Two fractions were analyzed: the pellet of exosomes at the end of the ultracentrifugation process and the supernatant containing exosomes of the pellet. As shown in Fig. 1, nanosized tracking analysis (NTA) revealed that the second fraction contained more exosomes picked at the expected size $(69 \mathrm{~nm})$. Protein analysis confirmed the NTA results (Fig 1C). Therefore, throughout the study we have used the second exosomal fragment. These results verified that Jurkat cells derived exosomes could be isolated. Subsequently, we exposed fibroblast cells to the isolated exosomes. Jurkat cells exosomes were labelled with FM 1-43 membrane fluorescent probe. The labeled exosomes were added to fibroblast cells in a dose dependent manner while control cells were treated with 
PBS only. FACS analysis was performed 24 hours afterwards. As demonstrated in Fig. 2, the FM 1-43 dye labeled fibroblast cells population was increased from $0.15 \%$ to $0.7 \%$ when $10^{11}$ exosomes were applied and to $45.19 \%$ when $10^{12}$ exosomes were used (Fig. 2C). Previous studies conducted in our laboratory showed that the optimal uptake time of these exosomes was $24 \mathrm{~h}$ (not shown).

\section{Jurkat cells derived exosomal hTERT partially induces the expression of CAF related markers}

In a previous study we showed that exposure of fibroblast cells to exosomes derived from Jurkat cells induced about 3.5-fold expression of hTERT in the recipient's previously telomerase negative cells [5]. These exosomes were previously isolated using a commercial kit. We repeated this experiment by using exosomes that were isolated by serial centrifugations and as shown in Fig. 3A the cargo of these exosomes contained the transcript of the hTERT gene. An increase of about 6.5-fold in the expression of the transcript of telomerase was detected 6 hours post exosomal exposure (Fig. 3B). As shown in our previous study, the hTERT transcript was translated into a fully active enzyme in the recipient's fibroblasts, thus modifying their phenotype from telomerase negative into telomerase positive cells.

Cancer cells' exosomes induce the expression of cancer associated fibroblasts (CAFs) in various experimental systems $[22,23]$. To understand whether some of CAFs markers are upregulated in response to telomerase activation, we exposed the fibroblast cells to Jurkat derived exosomes with or without the telomerase antagonist GRN163L. As demonstrated in Fig. 4A, exposed fibroblast cells exhibited a slight insignificant increase of $133 \%$ in aSMA levels after 24 hours. This elevation was not telomerase dependent as the inhibition of telomerase in the recipient fibroblasts did not reduce the levels of aSMA. However, 48h after exosomal exposure, the increase in aSMA was decreased when telomerase was inhibited, pointing to at least a partial dependency of the expression of aSMA in its activity.

A similar result was obtained when we analyzed the levels of vimentin. Twenty-four hours post exosomal exposure the levels of vimentin increased to $540 \%$ but were unchanged in response to the inhibition of telomerase. Forty-eight hours post exosomal exposure the levels of vimentin increased by $344 \%$. These levels decreased to $251 \%$ upon telomerase inhibition, again pointing to a partial dependency on telomerase activity (Fig. 4B). To gain more insight into possible connection of these CAF markers and telomerase, we employed the Advanced Network Annotation Tool (ANAT) software [15]. Based on databases of protein-protein interactions, ANAT connects a group of selected proteins (in our case- the identified CAF markers) to an "anchor" protein (human telomerase). The associations that are formed by ANAT are all statistically significant (compared to random input connections). This analysis identified a single pathway connecting telomerase and the CAF markers, Vimentin and aSMA, as follows: TERT- AKT1-Vimentin- GABARAP-1- aSMA.

These associations actually describe a direct biological link between telomerase, Vimentin and aSMA. 


\section{Fibroblast cells exposed to Jurkat cells derived exosomes secrete IL-6}

We next studied the effect of newly expressed telomerase in fibroblast cells on inflammation by assessing the expression of IL-6, as a key inflammatory cytokine. Two different concentrations of purified Jurkat cells derived exosomes were added to cells and equal volume of PBS was added to the control plates. The media was collected after 24,48 and 72 hours and subjected to human IL-6 immunoassay. As demonstrated in Fig. 5, when fibroblast cells were treated with the lower exosome concentration, IL-6 expression was 1.76-fold higher 24 hours post exosomal exposure compared to unexposed fibroblast cells. The effect was partially due to newly expressed telomerase since treated cells with telomerase inhibition exhibited a milder increase in IL-6 expression (1.37-fold higher then untreated fibroblast cells). The effect was abolished 48 hours after exosomal exposure. When the exosome concentration was higher (4-fold) the expression of IL- 6 increased by 2 fold after 24 hours compared to untreated fibroblasts. By 48 hours, the effect was slightly diminished and IL-6 expression was 1.88-fold higher in exosome treated fibroblast cells compared to untreated fibroblast cells. In each time point the effect was telomerase mediated to some degree since it was milder in treated fibroblasts with telomerase inhibition compared to exosomal exposed cells (Fig. 5). After 72 hours of exposure, the lower exosomal concentration did not affect the levels of IL- 6 by the recipient cells, but the higher exosomal concentration resulted in a similar level to that of the 48 hours effect (data not shown).

\section{Jurkat cells derived exosomes modulate the microRNA profile of pHFF cells}

In light of the study by Lassmann et al, describing the role of telomerase in microRNA upregulation [7], we sought to explore whether exosomal telomerase activity provides the recipient cells with a survival advantage, at least in part through the upregulation of specific microRNAs.

To test this hypothesis we exposed pHFF cells to Jurkat cells derived exosomes in the presence or absence of telomerase template antagonist, GRN163L. We and others have previously shown 80-90\% inhibition of the activity of the enzyme in response to that inhibitor $[24,25]$. We studied the profile of microRNA under six conditions:

- Jurkat cells cultured in exosome depleted media.

- pHFFscells, naturally devoid of telomerase activity and hTERT expression.

- pHFFs exposed to exosomes derived from Jurkat cells.

- pHFFs exposed to exosomesderived from Jurkat cellswith inhibited telomerase activity. For this purpose, pHFF cells were cultured in the presence of GRN163L before Jurkat cells derived exosomal exposure. 
- human foreskin fibroblasts (hFF) immortalized by the ectopic expression of hTERT- used as a positive control.

- Exosomes isolated from Jurkat cells grown in exosome depleted media.

Next, we compared the microRNA profile of the cells under the above experimental setting.

\section{The effect of Jurkat derived exosomes on microRNA expression of fibroblast cells}

When comparing fibroblasts with or without exosomes derived from Jurkat cells, the following microRNAs were significantly upregulated: hsa-miR-92a-3p, hsa-miR-128-3p, hsa-miR-342-3p, hsa-miR125b-1-3p, (Supp. Fig. 1A, Table 1).

We validated the expression of each of these microRNAs separately by Q-RT-PCR. As shown in Fig. 6, the expression of all four microRNAs was elevated upon exposure of fibroblasts to Jurkat cells derived exosomes.

\section{The effect of telomerase inhibition on the expression of microRNAs in fibroblasts exposed to Jurkat cells derived exosomes}

To understand the putative contribution of exosomal hTERT to microRNA expression in the recipient fibroblast cells, we compared the expression of microRNAs in fibroblasts exposed to exosomes with or without telomerase activity. As shown in Supp. Fig. 1B, the expression of four microRNAs was significantly upregulated and the expression of two microRNAs was downregulated as a result of the inhibition of telomerase activity in the recipient cells. These include: hsa-miR-491-5p hsa-miR-665 hsamiR-4730 hsa-miR-371b-5p that were upregulated and hsa-miR-30a-5p and hsa-miR-4284 whose expression decreased in response to Jurkat cells derived exosomes (Table 1).

Since miR- 342 was not upregulated as in cells that were exposed to Jurkat- derived exosomes upon telomerase inhibition and actually returned to base line levels and due to its known involvement in oncogenesis we selected it for the other parts of the study.

\section{The effect of telomerase inhibition on the expression of microRNAs in fibroblasts}

To gain more insight on microRNA expression resulting from exosomal telomerase we compared fibroblasts that were exposed to exosomes and inhibition of telomerase versus naïve fibroblasts. The expression of seven microRNAs was significantly changed when cells were put under these conditions. 
The following microRNAs were upregulated: hsa-miR-181a-5p; hsa-miR-378a-3p, hsa-miR-4730 and hsamiR-6132. Three microRNAs were downregulated in this setting: hsa-let-7e-5p, MID-20094 and hsa-miR664b-3p (Supp. Fig. 1C, Table 1).

\section{The effect of ectopic expression of telomerase on the microRNA profile of fibroblast cells}

We were able to compare the microRNA profile of fibroblast cells that were transfected with a plasmid expressing the catalytic subunit of telomerase, hTERT, expecting that this comparison will serve as a positive control. Supp. Fig. 1D shows the result of this analysis, pointing to other microRNAs that changed their expression in response to the overexpression of hTERT. These include two microRNAs whose expression was increased: hsa-miR-146a-5p and hsa-miR-490-5p. Three other microRNAs that decreased their expression are:hsa-miR-31-5p,MID-20524, hsa-miR-409-3p (Table 1).

\section{Profile of MicroRNAs that were packaged from Jurkat cells into their exosomes}

We compared the cellular microRNA content of Jurkat cells to their cognate exosome microRNAs (Supp. Fig. 1E). One hundred and twenty-two microRNAs were differentially expressed in the exosomes compared to the cells, 33 of these microRNAs levels were higher in the exosomes than in the cells, implying on a selective encapsulation of these miRs by exosomes. One of these higher expressed miRs was, miR-451a, implicated in several malignancies and proliferative processes [27]. This active packaging of microRNA molecules in exosomes may suggest the activity of a sorting mechanism which has functional implications.

\section{miR342 contributes to the proliferative rate and increases the S phase of the cell cycle of fibroblasts}

Taken together, the results of the microRNA expression analysis under the various conditions point to miR342 as a potential contributor to the survival advantage of exosomal exposed fibroblasts. To study its putative role in cell proliferation we overexpressed its open reading frame in fibroblasts (miR mimic 342, Fig. 7A) and assessed its expression in a time and dose dependent manner. As a negative control to miR mimic342 we used a plasmid containing a scrambled oligonucleotide. In addition, we transfected the fibroblast cells with an inhibitor of that miR and its negative control. Fibroblast cells with no plasmid (only transfection reagent) were used as another negative control. Thereafter, we measured the proliferation rate of the resulting fibroblasts by using the SRB assay. A difference of approximately 1.5fold of proliferation of pHFF cells containing ectopic miR-342 compared to that of the negative control 
was identified. The ectopic expression of miR-342 increased fibroblasts cell proliferation was compared to cells in which the scrambled oligo was expressed (Fig. 7B).

To understand whether the increase in proliferation stems from change in the cell cycle of the exposed cells, we used the same experimental system and assessed the cell cycle status by flow cytometry. As shown in Fig. 7C,D, exosomal exposure resulted in G1/S transition in the exposed cells. The S phase of the cell cycle of cells exposed to miR342 was increased by two-fold compared to the control untreated cells (Fig. 7C). Whereas $~ 13 \%$ of the cells transfected with miR342 were in the S phase of the cell cycle, in all other controls (including the negative control and the inhibitor related controls) about $6.3-7.4 \%$ of the cells were in the $S$ phase (Fig. 7D). All in all, these results suggest that miR-342 can increase the proliferation rate of fibroblasts cell, thus providing them with a survival advantage.

\section{miR342 involvement in biological processes related to survival}

To gain more insightabout the biological significance of miR342 we conducted several bioinformatic analyses. First, we looked at its target genes using ClueGO Cytoscape plugin software. As shown in Table 2 , some of its target categories include regulation of viable cell number, cell proliferation and cell death.

To further understand potential miR-342 targets involved in cell proliferation and cell cycle, we used several bioinformatic tools which identify putative miR binding sites based on the homology between the 3'UTR sequence of genes and miR sequences. The analysis was done by using TargetScan [11], miRBase [12], DIANAmiRPath [18], miRDB [15] and miRWalk [16]. From the results of this analysis we selected six genes that may be biologically relevant to cell proliferation. These include: RAS1, NKAP and RGS4. In addition, genes that are involved in epigenetic regulation may also serve as targets for miR342. These include the following: KMT2A-a lysine (K)-specific methyltransferase 2A, HAT1-histone acetyltransferase 1 , and the TRMT2A-a tRNA methyltransferase 2 homolog $A$. These proteins are all histone methylation modifying enzymes which mediate gene expression, genomic stability and mitosis $[27,28]$.

We have used the ANAT software here as well, to understand possible associations between the four selected miRs and telomerase. Since ANAT is a protein- protein based network, we first identified the protein regulators of each miR and then applied ANAT using telomerase as the anchor and the regulators as end nodes. The regulators are depicted in Table 3. Fig. 8 depict the networks formed by ANAT. These results point to different connectivity of telomerase and the protein regulators of the four miRs, reflecting a possible differential biological importance of each miR in our setting.

Finally we performed Gene Ontology (GO) terms analysis to shed more light on the putative functions of the four upregulated miRs in this setting (Supplemental Table 1).miRNAs were analyzed with DAVID functional Annotation tool to identify GO categories that are mostly represented in this subset.The results of this analysis demonstrated that the targets of the four miRs modulate the expression of genes that are 
implicated among other functions to mitotic cell cycle. Other gene products are related to protein, DNA and RNA metabolism.

Taken together, these analyses highlight both the putative biological connections between the miRs that were upregulated by exosomal telomerase and telomerase itself and the relevance of at least some of these miRs to proliferation processes.

\section{Discussion}

The importance of the cancer microenvironment in cancer progression and aggressiveness is well established. Tumor cells manipulate their microenvironment to support cancer by modulating stromal cells and the extra cellular matrix $[29,30]$. The intercellular communication between cancer cells and stromal cells, especially the one mediated by cancer-associated fibroblasts (CAFs) can be carried out among others, by exosomes [31]. Telomerase activity has been detected in approximately $90 \%$ of tumor samples of all types and is a central regulator of the hallmarks of cancer [32]. Telomerase importance in cancer biology motivated us to study its putative role in the crucial interactions between cancer cells and their surrounding microenvironment. In a previous study we showed that the transcript of telomerase is packaged in exosomes derived from cancer cell. This transcript is subsequently taken up by recipient cells, where it is translated into a fully active enzyme and its activity provides the cells with a survival advantage. In the current study we report two major biological processes that are regulated by exosomal telomerase in exposed fibroblasts: the upregulation of CAF associated markers and modulation of the microRNA landscape of these cells.

\section{The induction of CAF markers}

Numerous recent studies reported the transformation of fibroblasts to CAFs after exposure to cancer cells derived exosomes [33]. We examined the expression of two widely used CAFs markers in exosome treated fibroblast cells: aSMA and Vimentin. aSMA is the most widely used CAFs and myofibroblasts marker [34]. It is one of six different actin isoforms and was found to increase fibroblast cells' contractile ability. We found a slight telomerase dependency but an insignificant increase in aSMA levels in treated fibroblast cells 48 hours post Jurkat cells derived exosomal exposure. The lack of statistical significance may be attributed to the fact that the expression of that marker was assessed in the total fibroblast population and not in the sub-section of the cells that underwent CAFs transformation, or alternatively to the number of exosomes used in our setting which could potentially be scaled up. Another explanation may be attributed to the dependency of aSMA levels on the expression levels of TGF $\beta$ in the cancer cells derived exosomes to which the fibroblast cells were exposed as recently reported [35]. It may well be that our purified exosomes expressed a low level of TGFb.

Vimentin, a Type III intermediate filament, is expressed in mesenchymal cells, the origin of fibroblasts and confers mechanical stability and contributes to cell motility $[35,36]$. Exposure of the fibroblast cells to Jurkat cells derived exosomes elevated the levels of vimentin during 24 hours of exosomal exposure. 
This effect lasted by 48 hours and may be partially attributed to telomerase activity since cells with telomerase inhibition exhibited a milder effect. We also observed additional bands of vimentin after 48 hours of exosomal exposure that can arise from different isoforms of vimentin or posttranslational modifications $[37,38]$. The connection found by ANAT strengthens the experimental results and point to another extracurricular activity of telomerase.

IL-6 is a multifunctional cytokine that plays a central role in the regulation of inflammatory processes and is a prototype of an angiogenic related cytokine $[39,40]$. Many studies reported the secretion of IL- 6 from CAFs $[41,42]$ and its various tumor promoting downstream effects. In our setting, we found a clear trend of increase in IL-6 secretion levels from the exosomal exposed fibroblasts. Again, the effect was telomerase mediated to some degree since it was milder after inhibiting telomerase in the recipient fibroblasts by GRN163L. This effect can be mediated by TERT in a non-canonical pathway as IL-6 has been previously shown to be a direct target of telomerase activity [43].

\section{Exosomal hTERT regulates the biogenesis of microRNA in recipient fibroblasts}

The other part of our study describes the changes in miR expression in response to exosomal telomerase in these fibroblast cells.

miRNAs have been the focus of many biological studies in general and in the field of cancer research in particular as regulators of a large number of target genes, both as tumor suppressor or drivers. Given the fact that a single miRNA can target a large number of mRNA transcripts, aberrant expression of a set of miRNAs could have a significant effect on the cellular function by affecting multiple signaling pathways in the cells. Moreover, miRNAs are key regulators of the tumor-promoting functions and it is becoming clearer that cancer-directed changes regulate these miRNA based networks.

The rational for this part of our study was based on a recent report showing that telomerase activity was implicated in regulating the biogenesis of microRNAs [7]. To study the putative effect of exosomal telomerase on the expression of microRNAs in the recipient fibroblasts we exposed fibroblasts to Jurkat cells derived exosomes under the inhibition of telomerase activity in the recipient cells and analyzed the resulting microRNA landscape. We compared the expression of micrRNAs also to intact fibroblasts, to Jurkat cells and to fibroblast cells expressing hTERT. Using that analysis we identified four miRs that were significantly overexpressed in fibroblast cells exposed to exosomes containing hTERT compared to intact fibroblasts: miR-342-3p, miR-125b-1, miR-128-3p and miR-92a. By Q-PCR we validated the results of our analysis. These microRNAs were not expressed when telomerase activity was inhibited in the recipient cells and are involved in cancer related processes [44].

We also observed that the inhibition of hTERT was associated with dysregulation of miRNAs, which in turn could influence translation and function of over 600 gene targets. 
By comparing the expression of microRNAs in fibroblasts which stably expressed hTERT to the intact fibroblasts we identified a different set of microRNAs, with miR-146a showing the highest expression level in the presence of hTERT (20 fold). miR146 is implicated as a pleiotropic regulator of carcinogenesis [45]. The difference between the results of the two comparisons: that of exosomal hTERT versus that of ectopically expressed hTERT may stem from differences in the two experimental systems, for example, the expression levels of the gene.

\section{Active packaging of miRs in exosomes}

Interestingly, microRNA expression profile analysis of exosomal microRNAs derived from Jurkat cells identified some miRNAs that were expressed at higher levels in exosomes than in their parent cells, implying on an active packaging of molecules by exosomes and thus might have an important biological role in some setting. However, the mechanisms for selective packaging and release of exosomal microRNAs are currently unknown and may be affected by the malignant transformation process itself. As an example, we identified miR-451a, a regulator of tumor growth [46], which was highly expressed in Jurkat cells derived exosomes versus the parental donor cells.

\section{miR342}

In light of its higher expression and its biological role in carcinogenesis, miR-342 was chosen for the following parts of our study. To understand whether this miR is involved in the phenotypic changes obtained by exosomal telomerase [5] we the chosen miR was overexpressed in fibroblasts and we studied the putative effect on cell proliferation and cell cycle status. miR342 ectopic expression induced a 50\% increase in cell proliferation which is reflected by an increase of about two-fold in the S phase of the cell cycle of these cells suggesting that miR342 may act in the transition from G1 to S phase. These results are in accordance with the above-mentioned cancer related functions of that miR $[47,48]$. Of note, this miR was also expressed in the Jurkat cells but when analyzing the RNA cargo of their secreted exosomes we detected only a small, non-statistically significant amount of this miR. Since recipient cells in which telomerase was inhibited did not express miR342, we concluded that the activity of the enzyme is needed to activate the expression of miR342.

The results of our study may explain, at least in part, the survival effects provided by exosomal telomerase to the fibroblast recipient cells. Interestingly, the GO functions of the predicted target genes of the four miRs that were upregulated by exosomal telomerase, Toll Like Receptors (TLR) pathways, are in line with the increase in proliferation obtained in our setting (supplemental Table 1). The activation of TLR is implicated in the development and perpetuation of tumor cells by various mechanisms including the secretion of various cytokines promoting proliferation. The findings of CAF associated markers in response to exosomal telomerase at the first part of the study, especially the secretion of IL-6, fits well with the putative activation of TLR in inducing these cytokines secretion to promote tumor growth. 
When analyzing miR342 gene targets an enrichment of gene pathways related to proliferation was obtained. These include NKAP, RGS4 and RASA1 pathways. RASA1 acts as a tumor suppressor [49]; thereby its downregulation may promote cell proliferation through the RAS-RAF-MEK-ERK pathway [5052]. Similarly, the downregulation of NKAP may lead to cellular proliferation via the NF-kappa-B signaling pathway. Other predicted target genes of miR342 were implicated in epigenetic regulation. These include HAT1-histone acetyltransferase 1 and KMT2A-a lysine (K)-specific methyltransferase 2A, both involved in histone methylation implicated in malignant transformation [53]. Other pathways related to the targets of miR342 include the TGF-beta, the NF-KB and the p38/MAPK and cytokine-cytokine receptor interaction pathways, all highly related to the proliferative effect obtained by miR342.

\section{Telomerase non-canonical pathways}

In addition to telomeres maintenance, telomerase also harbors other biological roles which are defined as "extracurricular". Acting via NFKB and Wnt/ $\beta$-catenin pathways, these activities involve, among others, prevention of apoptosis, growth parameters and miRs biogenesis [54]. Here we show another side of the extracurricular activities of the enzyme, namely- the activation of miRs in exosome recipient cells. The interpretation of this result is supported by the analysis of ANAT, showing multiple connections between the four miR targets that were upregulated by the shuttle of exosomal telomerase and the enzyme itself.

The main limitation of current study is its in vitro artificial setting. In order to clarify the implications of our results, further study should measure exosome concentrations in cancer patients' sera and compare it to the exosome concentrations used in our study. Another difference between the in vitro and in vivo setting is the continuous nature of the process of secretion and uptake of exosomes in the human body versus a single dose exposure in vitro. Therefore, an in vivo study based on an animal model is needed. Clarifying these exosomal telomerase dependent microenvironmental relationships may be exploited in the future for the development of new rationalized drugs against many types of cancer.

In summary, our study shows that hTERT exosomes alter the phenotype and the microRNA transcription profile of the recipient fibroblasts. Specifically, two CAF related markers were upregulated, one cytokine was secreted and four microRNAs including miR342. These changes may promote the exposed fibroblasts to support cancer progression by providing them with a survival advantage through exosomal telomerase.

\section{References}

1. Quail DF; Joyce JA. Microenvironmental regulation of tumor progression and metastasis. Nat Med. 2013 19, 1423-37. doi: 10.1038/nm.3394.

2. Whiteside TL. The tumor microenvironment and its role in promoting tumor growth. Oncogene. 2008, 27, 5904-12. doi: 10.1038/onc.2008.271. 
3. Ramachandran S; Palanisamy V. Horizontal transfer of RNAs: exosomes as mediators of intercellular communication. Wiley Interdiscip Rev RNA. 2012 3, 286-93. doi: 10.1002/wrna.115.

4. Bryan TM; Cech TR. Telomerase and the maintenance of chromosome ends. Curr Opin Cell Biol. 1999 11, 18-24. doi: 10.1016/S0955-0674(99)80043-X.

5. Gutkin A; Uziel O; Beery E; Nordenberg J; Pinchasi M; Goldvaser H; Henick S; Goldberg M; Lahav M. Tumor cells derived exosomes contain hTERT mRNA and transform nonmalignant fibroblasts into telomerase positive cells. Oncotarget. 2016 7, 59173-59188. doi: 10.18632/oncotarget.10384.

6. Jaiswal RK; Kumar P; Yadava PK. Telomerase and its extracurricular activities. Cell Mol Biol Lett. 2013 18, 538-54. doi: 10.2478/s11658-013-0105-0.

7. Lassmann T; Maida Y; Tomaru Y; Yasukawa M; Ando Y; Kojima M; Kasim V; Simon C; Daub CO; Carninci P; Hayashizaki Y; Masutomi K. Telomerase reverse transcriptase regulates microRNAs. Int J Mol Sci. 2015 16, 1192-208. doi: 10.3390/ijms16011192.

8. Hahn WC; Counter CM; Lundberg AS; Beijersbergen RL; Brooks MW; Weinberg RA. Creation of human tumour cells with defined genetic elements. Nature. 1999 400, 464-8. doi: 10.1038/22780.9.

9. Dragovic RA; Gardiner C; Brooks AS; Tannetta DS; Ferguson DJ; Hole P; Carr B; Redman CW; Harris AL; Dobson PJ; Harrison P; Sargent IL. Sizing and phenotyping of cellular vesicles using Nanoparticle Tracking Analysis. Nanomedicine. 2011 7, 780-8. doi: 10.1016/j.nano.2011.04.003.

10. http:/www.targetscan.org

11. https://www.bmh.manchester.ac.uk/research/impact/mirbase/

12. http:/www.microrna.org

13. http://diana.cslab.ece.ntua.gr

14. http://mirdb.org

15. http://www.ma.uni-eidelberg.de/aps/zmf/mirwalk

16. http://genie.weizmann.ac.il

17. http://www.microrna.gr/miRPathv2

18. http://david.abcc.ncifcrf.gov/

19. Bindea G; Mlecnik B; Hackl H; Charoentong P; Tosolini M; Kirilovsky A; Fridman WH; Pagès F; Trajanoski Z; Galon J. ClueGO: a Cytoscape plug-in to decipher functionally grouped gene ontology and pathway annotation networks. Bioinformatics. 2009 25, 1091-3. doi:

10.1093/bioinformatics/btp101.

20. Yosef N; Zalckvar E; Rubinstein AD; Homilius M; Atias N; Vardi L; Berman I; Zur H; Kimchi A; Ruppin E; Sharan R. ANAT: a tool for constructing and analyzing functional protein networks. Sci Signal. 2011 4, pl1. doi: 10.1126/scisignal.2001935.

21. Ringuette Goulet C; Bernard G; Tremblay S; Chabaud S; Bolduc S; Pouliot F. Exosomes Induce Fibroblast Differentiation into Cancer-Associated Fibroblasts through TGF $\beta$ Signaling. Mol Cancer Res. 2018 16, 1196-1204. doi: 10.1158/1541-7786.MCR-17-0784. 
22. Gu J; Qian H; Shen L; Zhang X; Zhu W; Huang L; Yan Y; Mao F; Zhao C; Shi Y; Xu W. Gastric cancer exosomes trigger differentiation of umbilical cord derived mesenchymal stem cells to carcinomaassociated fibroblasts through TGF- $\beta /$ Smad pathway. PLoS One. 2012 7, e52465. doi: 10.1371/journal.pone.0052465

23. Shammas MA; Koley H; Bertheau RC; Neri P; Fulciniti M; Tassone P; Blotta S; Protopopov A; Mitsiades C; Batchu RB; Anderson KC; Chin A; Gryaznov S; Munshi NC. Telomerase inhibitor GRN163L inhibits myeloma cell growth in vitro and in vivo. Leukemia. 2008 22, 1410-8. doi: 10.1038/leu.2008.81. Epub 2008 May 1.

24. Uziel O; Beery E; Dronichev V; Samocha K; Gryaznov S; Weiss L; Slavin S; Kushnir M; Nordenberg Y; Rabinowitz C; Rinkevich B; Zehavi T; Lahav M. Telomere shortening sensitizes cancer cells to selected cytotoxic agents: in vitro and in vivo studies and putative mechanisms. PLoS One. 2010 5, e9132. doi: 10.1371/journal.pone.0009132.

25. Su Z; Ni L; Yu W; Yu Z; Chen D; Zhang E; Li Y; Wang Y; Li X; Yang S; Gui Y; Lai Y; Ye J. MicroRNA-451a is associated with cell proliferation, migration and apoptosis in renal cell carcinoma. Mol Med Rep. 2015 11, 2248-54. doi: 10.3892/mmr.2014.2957.

26. Del Rizzo PA; Trievel RC. Substrate and product specificities of SET domain methyltransferases. Epigenetics. 2011 6, 1059-67. doi: 10.4161/epi.6.9.16069. Epub 2011 Sep 1.

27. Audia JE; Campbell RM. Histone Modifications and Cancer. Cold Spring Harb Perspect Biol. 20168 , a019521. doi: 10.1101/cshperspect.a019521.

28. Bissell MJ; Hines WC. Why don't we get more cancer? A proposed role of the microenvironment in restraining cancer progression. Nat Med. 2011 17, 320-9. doi: 10.1038/nm.2328.

29. Joyce JA; Pollard JW. Microenvironmental regulation of metastasis. Nat Rev Cancer. 2009 9, 239-52. doi: 10.1038/nrc2618. Epub 2008 Mar 12.

30. Kenny PA; Lee GY; Bissell MJ. Targeting the tumor microenvironment. Front Biosci. 2007 12, 3468-74. doi: $10.2741 / 2327$.

31. Kim NW; Piatyszek MA; Prowse KR; Harley CB; West MD; Ho PL; Coviello GM; Wright WE; Weinrich SL; Shay JW. Specific association of human telomerase activity with immortal cells and cancer. Science. 1994 266, 2011-5. doi: 10.1126/science.7605428.

32. Guo W; Gao Y; Li N; Shao F; Wang C; Wang P; Yang Z; Li R; He J. Exosomes: New players in cancer. Oncol Rep. 2017 38, 665-675. doi: 10.3892/or.2017.5714.

33. Shiga K; Hara M; Nagasaki T; Sato T; Takahashi H; Takeyama H. Cancer-Associated Fibroblasts: Their Characteristics and Their Roles in Tumor Growth. Cancers (Basel). 2015, 72443-58. doi: 10.3390/cancers7040902.

34. Webber J; Steadman R; Mason MD; Tabi Z; Clayton A. Cancer exosomes trigger fibroblast to myofibroblast differentiation. Cancer Res. 2010 70, 9621-30. doi: 10.1158/0008-5472.CAN-10-1722.

35. Satelli A; Li S. Vimentin in cancer and its potential as a molecular target for cancer therapy. Cell Mol Life Sci. 2011 68, 3033-46. doi: 10.1007/s00018-011-0735-1. 
36. Eckes B; Dogic D; Colucci-Guyon E; Wang N; Maniotis A; Ingber D, Merckling A, Langa F, Aumailley M, Delouvée A, Koteliansky V, Babinet C, Krieg T. Impaired mechanical stability, migration and contractile capacity in vimentin-deficient fibroblasts. J Cell Sci. 1998 111, 1897-907.

37. Van Steendam K; Tilleman K; De Ceuleneer M; De Keyser F; Elewaut D; Deforce D. Citrullinated vimentin as an important antigen in immune complexes from synovial fluid of rheumatoid arthritis patients with antibodies against citrullinated proteins. Arthritis Res Ther. 2010, 12, R132. doi: 10.1186/ar3070.

38. Buchmaier BS; Bibi A; Müller GA; Dihazi GH; Eltoweissy M; Kruegel J; Dihazi H. Renal cells express different forms of vimentin: the independent expression alteration of these forms is important in cell resistance to osmotic stress and apoptosis. PLoS One. 2013,8 :e68301. doi: 10.1371/journal.pone.0068301.

39. Ghosh A; Saginc G; Leow SC; Khattar E; Shin EM; Yan TD; Wong M; Zhang Z, Li G, Sung WK, Zhou J, Chng WJ, Li S, Liu E, Tergaonkar V. Telomerase directly regulates NF-KB-dependent transcription. Nat Cell Biol. 2012 14, 1270-81. doi: 10.1038/ncb2621.

40. Wei LH; Kuo ML; Chen CA; Chou CH; Lai KB; Lee CN; Hsieh CY. Interleukin-6 promotes cervical tumor growth by VEGF-dependent angiogenesis via a STAT3 pathway. Oncogene. 2003 22, 1517-27. doi: 10.1038/sj.onc. 1206226.

41. Wu X; Tao P; Zhou Q; Li J; Yu Z; Wang X; Li J; Li C; Yan M; Zhu Z; Liu B; Su L. IL-6 secreted by cancerassociated fibroblasts promotes epithelial-mesenchymal transition and metastasis of gastric cancer via JAK2/STAT3 signaling pathway. Oncotarget. 2017 8, 20741-20750. doi:

10.18632/oncotarget.15119.

42. Shintani Y; Fujiwara A; Kimura T; Kawamura T; Funaki S; Minami M; Okumura M. IL-6 Secreted from Cancer-Associated Fibroblasts Mediates Chemoresistance in NSCLC by Increasing EpithelialMesenchymal Transition Signaling. J Thorac Oncol. 2016 11, 1482-92. doi: 10.1016/j.jtho.2016.05.025.

43. Trino S; Lamorte D; Caivano A; Laurenzana I; Tagliaferri D; Falco G; Del Vecchio L; Musto P; De Luca L. MicroRNAs as New Biomarkers for Diagnosis and Prognosis, and as Potential Therapeutic Targets in Acute Myeloid Leukemia. Int J Mol Sci. 2018, 19, :460. doi: 10.3390/ijms19020460.

44. Min SK; Jung SY; Kang HK; Park SA; Lee JH; Kim MJ; Min BM. Functional diversity of miR-146a-5p and TRAF6 in normal and oral cancer cells. Int J Oncol. 2017 51, 1541-1552. doi: 10.3892/ijo.2017.4124.

45. Bai H; Wu S. miR-451: A Novel Biomarker and Potential Therapeutic Target for Cancer. Onco Targets Ther. 2019 12, 11069-11082. doi: 10.2147/OTT.S230963.

46. Tao K; Yang J; Guo Z; Hu Y; Sheng H; Gao H; Yu H. Prognostic value of miR-221-3p, miR-342-3p and miR-491-5p expression in colon cancer. Am J Transl Res. 2014 6, 391-401.

47. He YJ; Wu JZ; Ji MH; Ma T; Qiao EQ; Ma R; Tang JH. miR-342 is associated with estrogen receptor-a expression and response to tamoxifen in breast cancer. Exp Ther Med. 2013 5, 813-818. doi: 10.3892/etm.2013.915. 
48. Hu X; Stern HM; Ge L; O'Brien C; Haydu L; Honchell CD; Haverty PM; Peters BA; Wu TD; Amler LC; Chant J; Stokoe D; Lackner MR; Cavet G. Genetic alterations and oncogenic pathways associated with breast cancer subtypes. Mol Cancer Res. 2009 7, 511-22. doi: 10.1158/1541-7786.MCR-08-0107

49. Donovan S; Shannon KM; Bollag G. GTPase activating proteins: critical regulators of intracellular signaling. Biochim Biophys Acta. 2002 1602, 23-45. doi: 10.1016/s0304-419x(01)00041-5.

50. Denhardt DT. Signal-transducing protein phosphorylation cascades mediated by Ras/Rho proteins in the mammalian cell: the potential for multiplex signalling. Biochem J. 1996 318 (Pt 3)(Pt 3):729-47. doi: 10.1042/bj3180729.

51. Downward J. Targeting RAS signalling pathways in cancer therapy. Nat Rev Cancer. 2003 3, 11-22. doi: $10.1038 / \operatorname{nrc} 969$.

52. Chen $\mathrm{F}_{\text {; }}$ Kan H; Castranova V. "Methylation of Lysine 9 of Histone H3: Role of Heterochromatin Modulation and Tumorigenesis". 2010. In Tollefsbol TO. Handbook of Epigenetics: The New Molecular and Medical Genetics. Boston: Academic Press. pp. 149-157.

53. Ding D; Xi P; Zhou J; Wang M; Cong YS. Human telomerase reverse transcriptase regulates MMP expression independently of telomerase activity via NF-kB-dependent transcription. FASEB J. 2013 27, 4375-83. doi: 10.1096/fj.13-230904.

\section{Tables}

Due to technical limitations, tables are only available as a download in the Supplemental Files section.

\section{Figures}



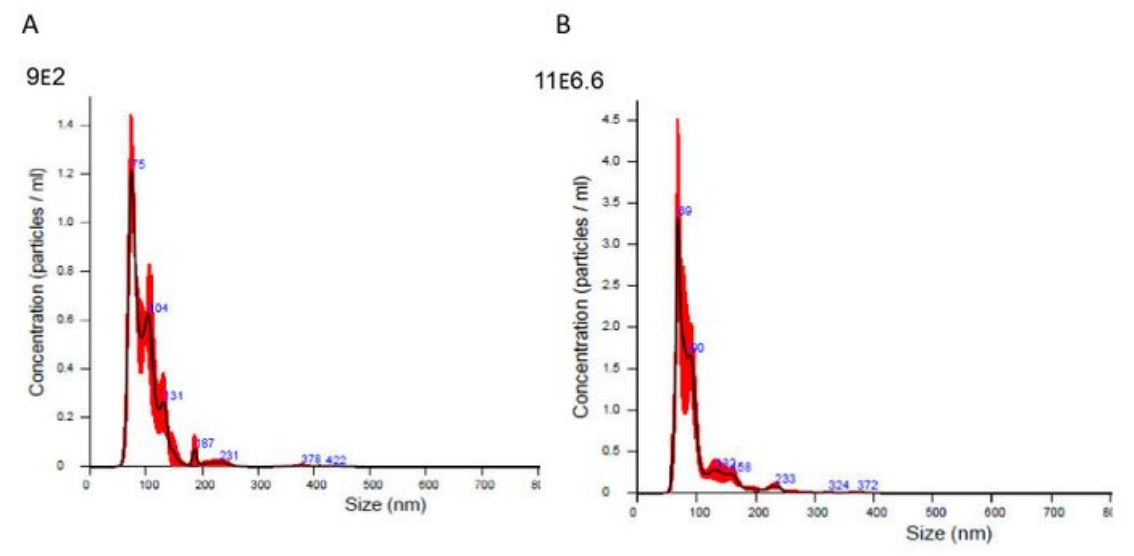

c

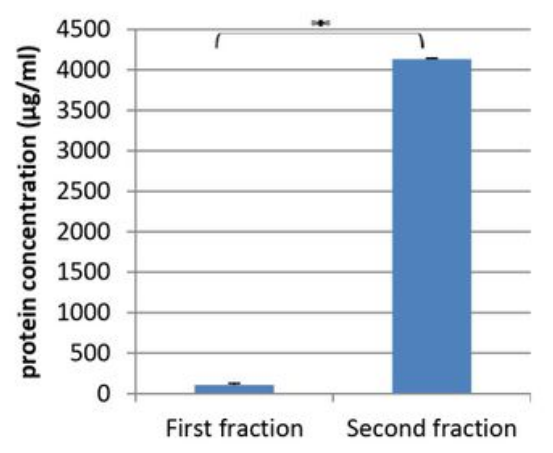

\section{Figure 1}

Quantification of the Jurkat cells exosomes purification. Jurkat cells exosomes were purified from two fractions. The two fractions were quantified using the Nano- Sight tracking analysis (NTA). Represented images of the quantification of the first fraction (using dilution factor 1:200) (A) and the quantification of the second fraction (using dilution factor 1:6600) (B) are shown. (C) Quantification of total protein concentration of the two fractions of purified Jurkat cells exosomes. The graphs values represent mean concentration \pm S.E of three independent experiments. *indicates $P V<0.001$. D. Examples of single exosomes images captured by EM. 
A

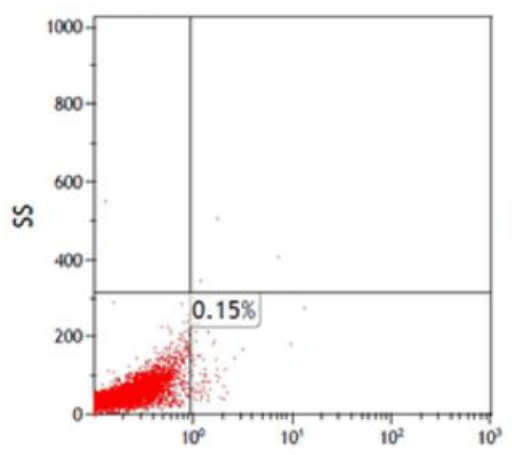

C

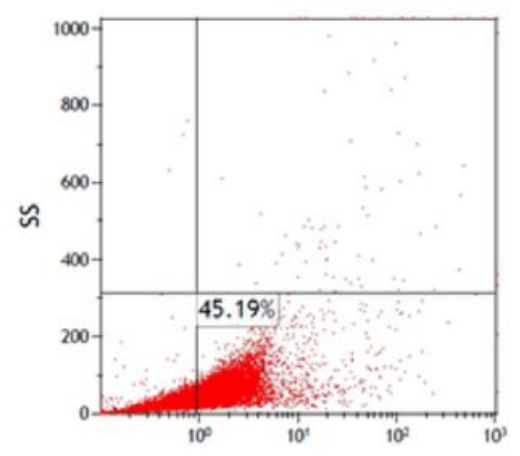

B
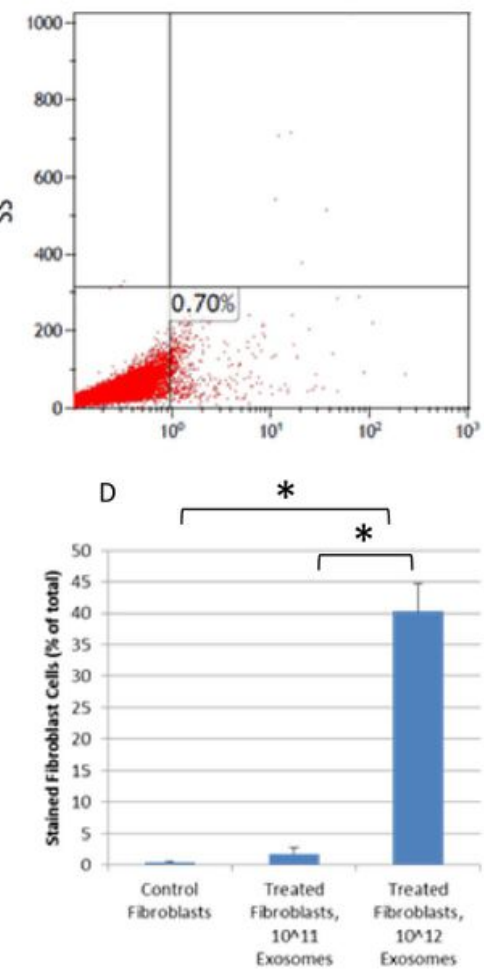

\section{Figure 2}

Jurkat cells derived exosomes uptake by fibroblast cells. Jurkat cells derived exosomes were labeled using FM 1-43 membrane dye. Fibroblast cells were treated with several exosome concentrations for 24 hours. Exosomal uptake was determined using FACS analysis. $X$ axis: percent of fibroblast cells labeled with FM 1-43. (A) Control fibroblast cells. (B) Fibroblast cells treated with 1011 exosomes. (C) Fibroblast cells treated with 1012 exosomes. Represented images are shown. (D) Quantification of exosomal uptake. The graphs values represent mean (\%) \pm S.E of three independent experiments. * indicates PV< 0.05 . 
A

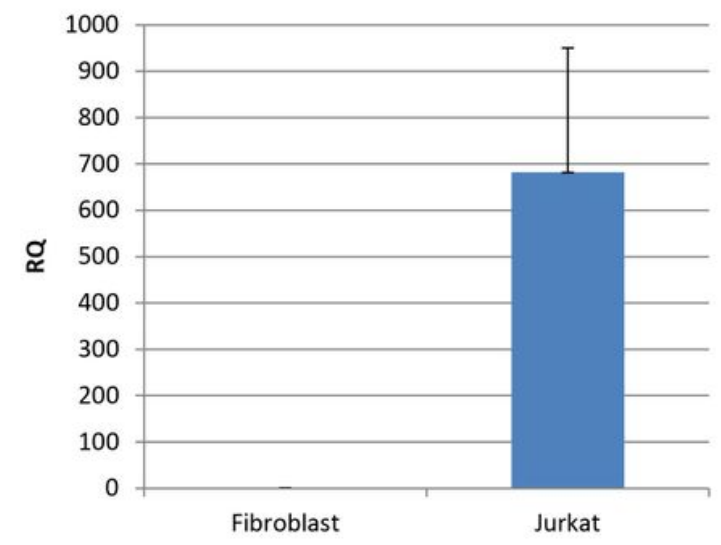

B

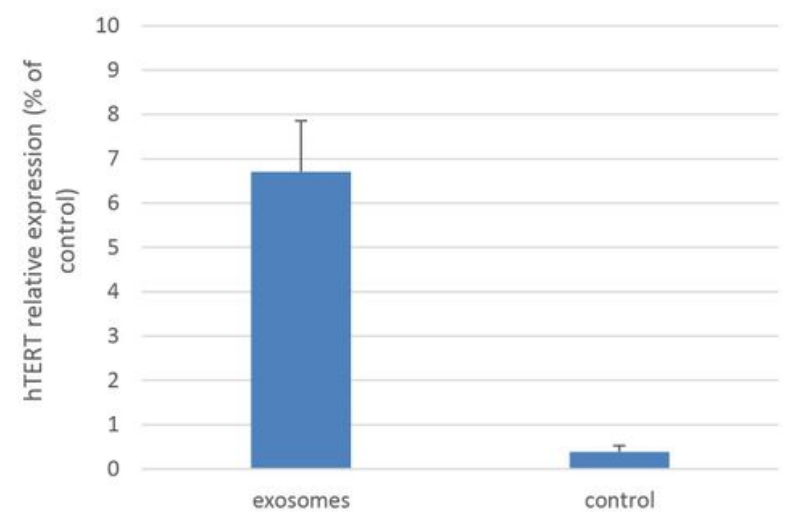

\section{Figure 3}

hTERT expression in Jurkat cells exosomes and in subsequent fibroblast cells. A. Exosomal RNA was isolated from three different exosome isolations. hTERT and HPRT-1 as a control gene were amplified by Real-Time PCR. The $\mathrm{Y}$ axis represents the normalized hTERT expression level in Jurkat cells exosomes compared to control fibroblast cells exosomes. For calculation needs, a threshold of $\mathrm{Ct} 50$ was used as the "undetectable" hTERT expression in fibroblast cells. The bars represent average hTERT expression of each sample \pm S.E of three independent experiments. B. $3 \times 105 \mathrm{pHFF}$ cells $/ \mathrm{ml}$ cells were subjected to Jurkat-derived exosomes for $6 \mathrm{~h}$. The cells were harvested and the expression of the hTERT mRNA was carried out by quantitative real time PCR. Each column represents mean (\%) \pm S.E of three independent experiments. Exosomes: cells exposed to Jurkat derived exosomes, control-cells exposed to PBS only. 


\section{FIG 4A}

A

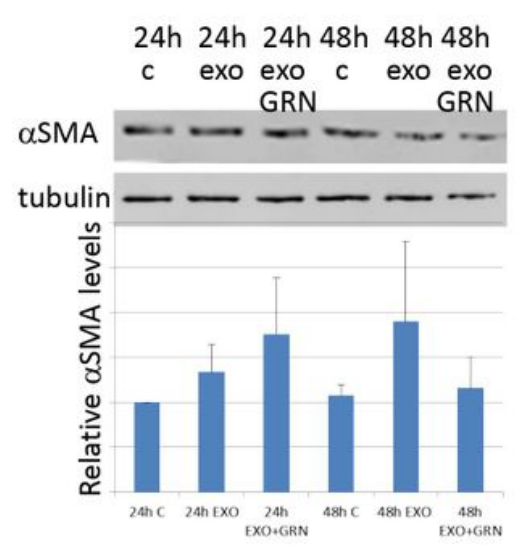

B

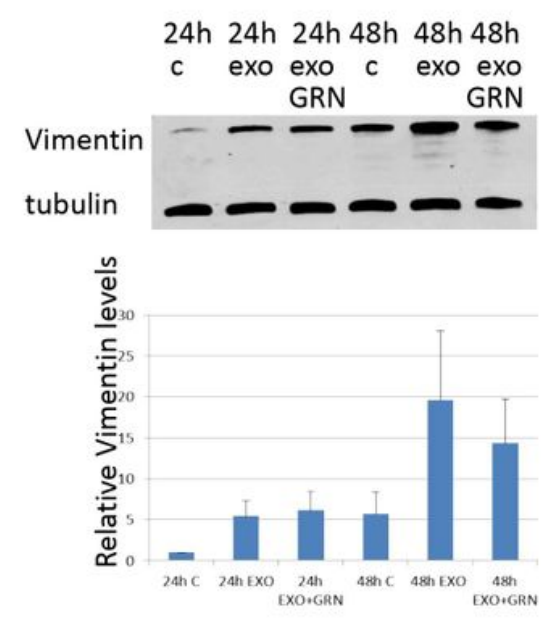

\section{Figure 4}

CAFs markers levels in exosome exposed fibroblast cells. Fibroblast cells were exposed to Jurkat cells derived exosomes (Exo), to telomerase inhibitor (GRN) or to Jurkat-derived exosomes and telomerase inhibitor (Exo+GRN) for 24 and 48 hours. Cells were harvested, lysed and proteins were extracted. $25 \mu \mathrm{g}$ of protein lysate was subjected to Western blot analysis to determine possible alterations in the expression levels of the CAFs markers: (A) aSMA and (B) Vimentin. Upper panel: example of one representative Western blot. Lower panel: Quantification of the cellular levels of the analyzed proteins. Each column represents mean $(\%) \pm$ S.E of three independent experiments. 


\section{FIG 5}

॥ $1.6^{*} 10^{\wedge} 12$ exosomes $/ \mathrm{ml}$

- 4*10^11 exosomes $/ \mathrm{ml}$

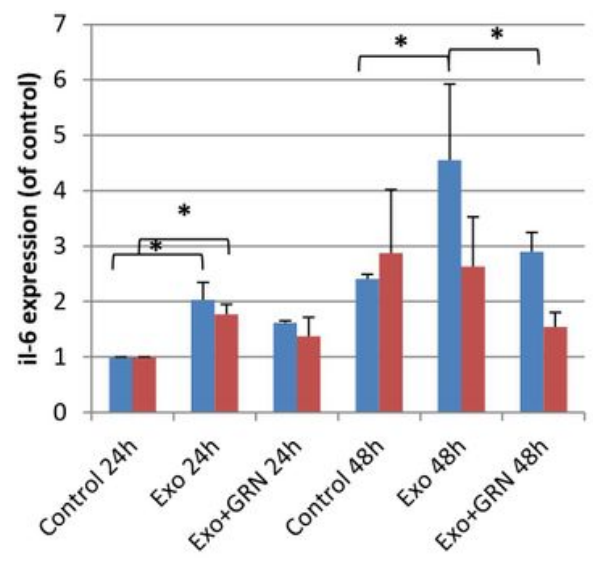

Figure 5

IL-6 secretion from exosome treated fibroblast cells. Fibroblast cells were exposed to Jurkat cells derived exosomes (Exo) in two different concentrations, to telomerase inhibitor (GRN) or to Jurkat-derived exosomes and telomerase inhibitor (Exo+GRN) for 24 and 48 hours. Media was collected and IL-6 protein levels in the supernatants were measured by ELISA. Each column represents mean (\%) \pm S.E of at least two independent experiments. *Indicates PV $<0.05$. 


\section{FIG 6}

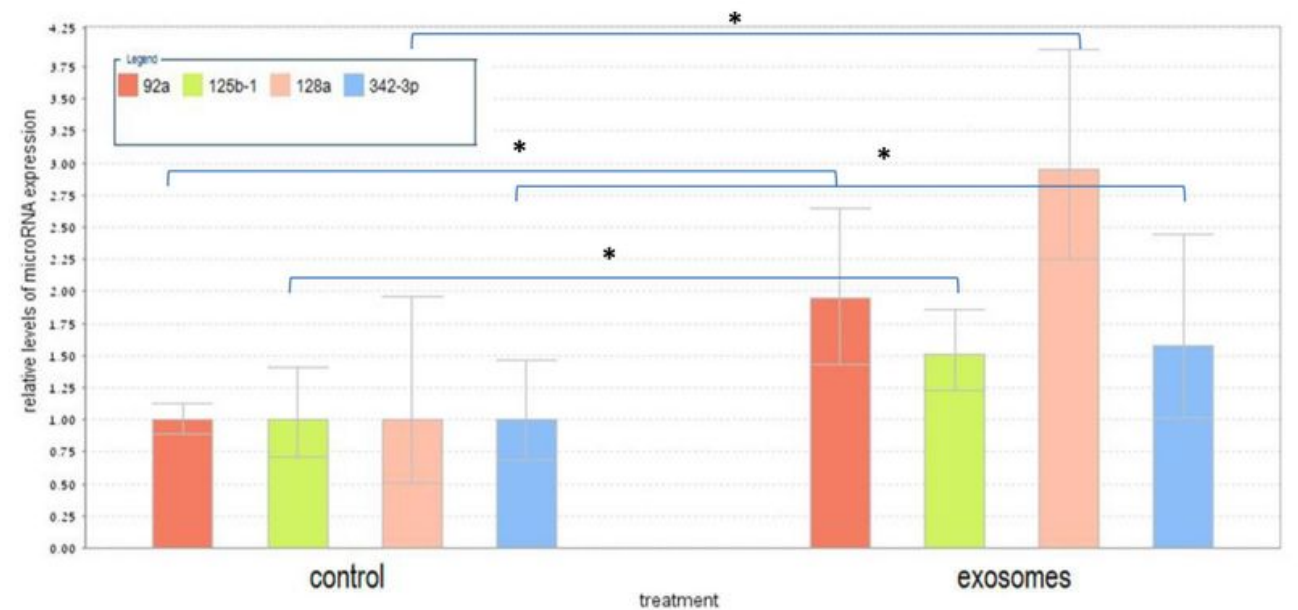

\section{Figure 6}

Analysis of relative microRNA expression after Jurkat derived exosomal exposure. A. Identification of miRNAs which were differentially expressed in pHFF cells incubated with exosomes from Jurkat cells ( $y$ axis) versus pHFF cells with no exosomal exposure (x-axis). Each blue cross represents a miRNA; Grey crosses are probes not tested in the analyses (e.g. control sequences or low expression). Each cross signifies the median expression of a probe in the two groups; Red circles: miRs with fold-change $>2$, $\mathrm{p}<0.05 \mathrm{~B}$. Q-Real-time PCR validation of the microarray data. Expression levels of miRNAs were measured by quantitative RT-PCR (Q-RT-PCR) in a subset of 4 samples. Cycle threshold values for each miRNA were normalized versus those of the housekeeping small RNA U3 gene. Q-RT-PCR showed that all four selected miRNAs display higher expression in the relevant samples after Jurkat cells derived exosomal exposure. Four miRNAs expression were correlated with the microarray results. Quantitative RT-PCR data are represented as the mean $\pm S D ; n=3$. The statistical significance was ${ }^{*} p<0.05$ as measured using the Student-t test. C. Correlation plot between miRNA expression in pHFFs incubated with exosomes + telomerase inhibitor GRN163 (y-axis) vs. pHFF incubated with exosomes only (x-axis). Upregulated miRs were enriched for G-rich miRs (technical bias), so additional filters were used for this comparison. In black 
circled yellow: fold-change $>2$, p-value $<0.05$ and miRs that are not G-rich. D. Correlation plot between miRNA expression in pHFFs incubated with exosomes + telomerase inhibitor GRN163 (y-axis) vs. pHFF alone (x-axis). Diagonal lines demarcate 1.5-fold difference levels in expression between the two groups; In blacked circled yellow: $p<0.05$ with the largest fold-change (fold-change $>5$ ). Grey crosses are probes not tested in analyses (e.g. control sequences or low expression). E. miRNA expression in pHFF cells transfected with the hTERT gene (y-axis) vs pHFF wild type cells ( $x$-axis). Bold red circles depict a differential change of $>2$. F. miRNA expression in Jurkat cells ( $y$-axis) vs Jurkat cells derived exosomes ( $x-$ axis). miRs that were significantly differentially expressed are marked in Blue.

\section{FIG 7A}
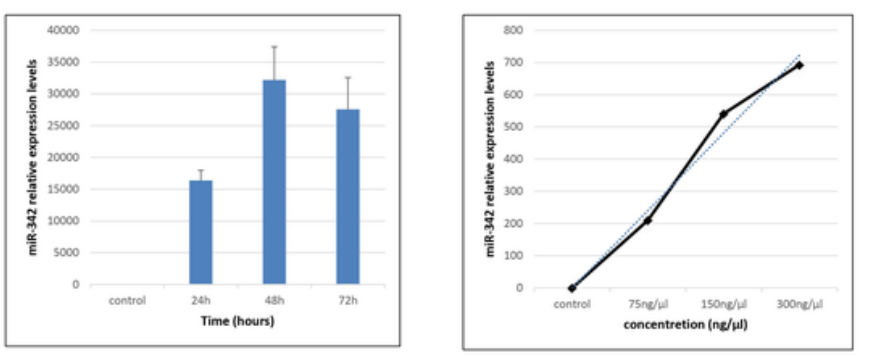

FIG 7C
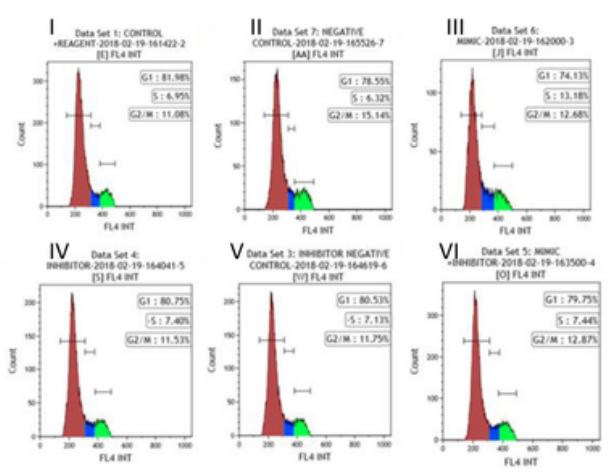

FIG 7D

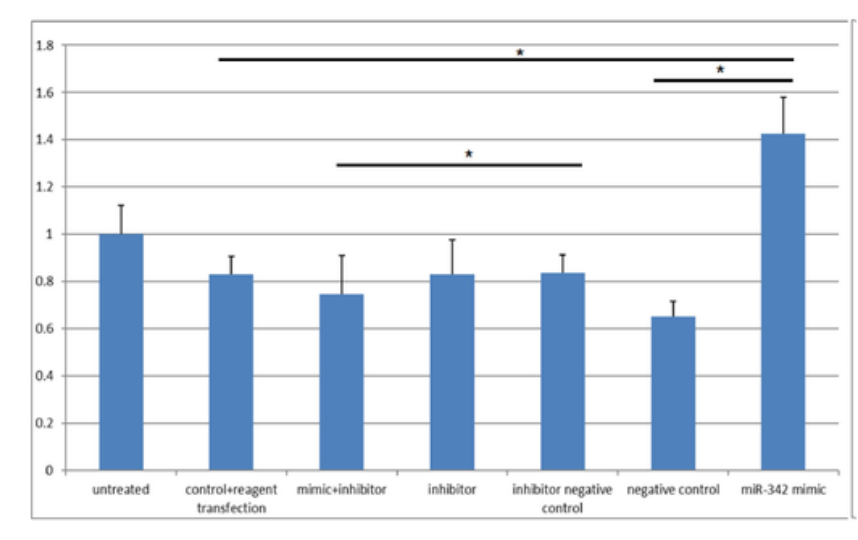

FIG 7B

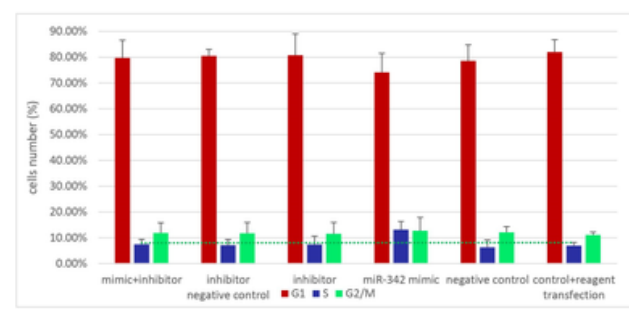

\section{Figure 7}

miR342 provides fibroblasts cells with a survival advantage A. Transfection of pHFF cells with miR342. Left panel: Q-RT-PCR analysis of the relative expression levels of miR-342 after its mimic transfection. The standard Taqman MicroRNA Assays was followed to examine performance of RNU6B (snRNA) endogenous control candidates served as an internal reference. The data summarizes three experiments and expressed as mean \pm SD for three experiments. right Panel: Q- RT-PCR analysis of the relative expression levels of miR342 mimic in the pHFF cells after treatment with various concentrations of that miR-342 mimic (75 ng/ $\mu \mathrm{l}, 150 \mathrm{ng} / \mu \mathrm{l}, 300 \mathrm{ng} / \mu \mathrm{l})$ B. MiR-342 overexpression increases cellular proliferation. 96 hours after $150 \mathrm{ng} / \mu \mathrm{LmiR}$ mimic 342 and its various controls transfection, we observed a 
statistically significant increase in the growth of miR-342 overexpressing cells in comparison to cells treated by negative control, untreated fibroblast, or fibroblast + reagent transfection $(p<0.05)$ By using the $\mathrm{SRC}$ assay. The data summarizes three independent experiments and expressed as mean \pm SD. C. The cell cycle status of pHFF cells in reponse to the ectopic expression of miR342. The DNA content of the cells was measured by flow cytometry. Cells were processed by standard methods using propidium iodide staining of DNA. (I) pHFF cell-cycle profile of cells transfected with transfection reagent. (II) pHFF cellcycle profile of cells transfected with negative control. (III) pHFF cell-cycle profile of cells transfected with miR-342 mimic (IV) pHFF cell-cycle profile of cells transfected with miR-342 inhibitor (V) pHFF cell-cycle profile of cells transfected with inhibitor negative control $(\mathrm{VI})$ pHFF cell-cycle profile of cells transfected with miR-342 mimic+ miR342 inhibitor. D. Quantification of 7C. Graph displaying differences in cell cycle phases, as determined by FACS analysis, between normal and miR-342 expressing pHFF cells. The data summarizes three independent experiments and expressed as mean \pm SD.

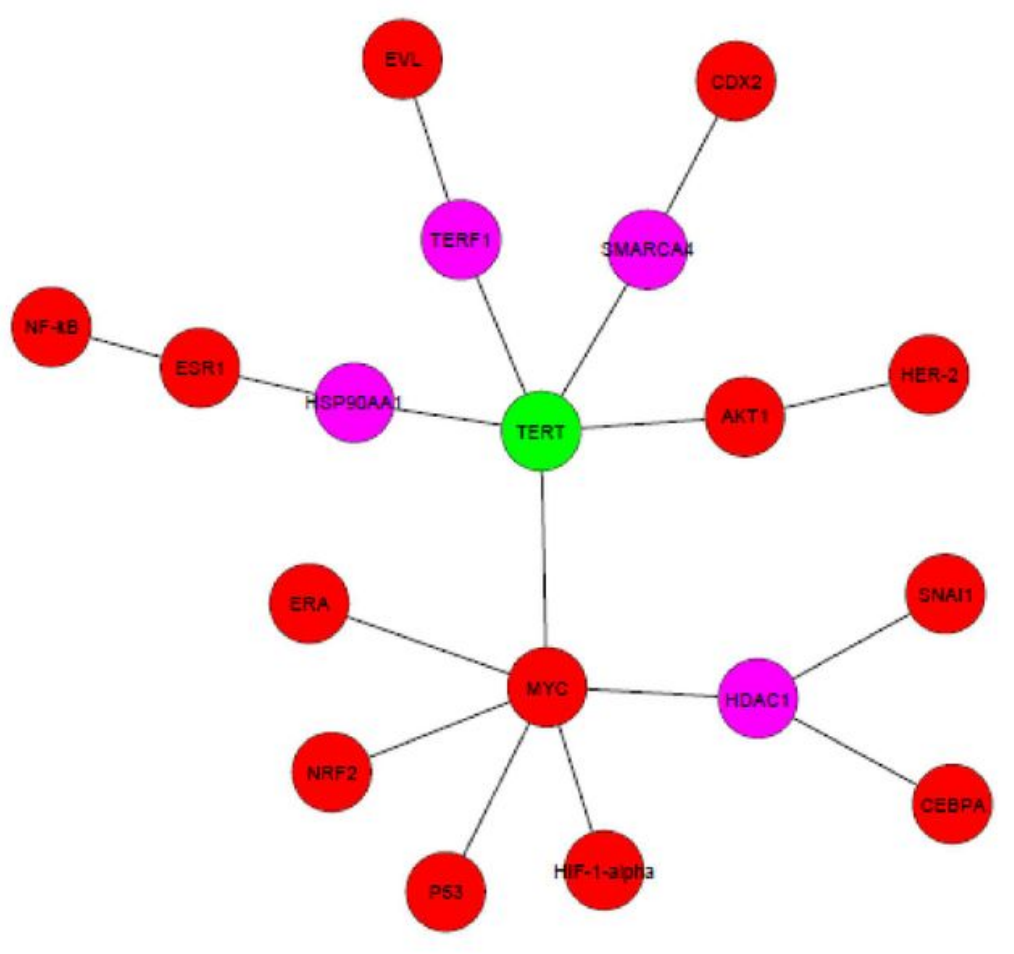

\section{Figure 8}

ANAT output of four miRNA target proteins and telomerase. Pink circles represent proteins identified by ANAT. Brown circles are the targets of the miRs predicted by miRDB. The green circle refers to the anchor 
telomerase (hTERT). A. Interaction map showing the connections of telomerase (hTERT) and genes targeted by the miR-342. B. Interaction map showing the connections of telomerase (hTERT) and genes targeted by the miR-92-a. C. Interaction map showing the connections of telomerase (hTERT) and genes targeted by the miR-125-b. D. Interaction map showing the connections of telomerase (hTERT) and genes targeted by the miR-128.

\section{Supplementary Files}

This is a list of supplementary files associated with this preprint. Click to download.

- SuppTable1.docx

- suppfigures.pdf

- Tab1.jpg

- Tab2.jpg

- Tab3.jpg 